The aluminum film can also be made much thicker. In this way more of the excitation volume can be filled with aluminum and copper, and less with silicon. This is a good solution where practical, but in our case the engineers wanted to sample on actual production wafers and weren't willing to make the films $3 \mathrm{ml}$ crons thick.

Finally, you can collect the x-rays for a longer time. As long as the counts in the peak are accumulating faster than the square root of the background counts, eventually the peak will be distinguishable. It can be quite frustrating, however, to find yourself in the opposite condition where you have collected an $x$-ray spectrum for an hour or two and there is still no peak! Don't be deceived, though - just because you don't see a peak doesn't mean that the element is not there!

(By the way, for logistical reasons we finally established the analytical method to be $x$-ray fluorescence spectromety utilizing an energy-dispersive XRF instrument that was already located on the production floor near the aluminum evaporators.)

\section{Budget Constraints?}

\$ Lease or rent your new SEM, TEM, EDS or other laboratory equipment with buyout options.

\$Plans are easily tailored to your budgetary needs.

\$Our fast approval, low rates, and pleasant service will please you.

\section{For more information call: \\ Greg Smith at (800)700-6680 \\ Concord Funding Group 26 Main Street \\ Concord, MA 01742}

\section{An Effective, Cost-Free Holder/Desiccator} for Storage of SEM Stubs

Glenn Walker and Jerry Hartenburg, Eastern Michigan University, and Chris Edwards, University of Michigan

Two of the aggravations of effectively managing an EM unit are cost containment and effective storage of large numbers of specimens. For the past several years we have enjoyed the benefits of a stub-storage box (for pin-type stubs) available to microscopists without cost. These boxes, which held micro-pipette tips, are regularly discarded by molecular biology and biochemistry labs. In fact, since colleagues have recognized that we use their spent plastic boxes, we have been inundated with boxes oniginally slated for recycling. While several types of these boxes are available, most measure approximately twelve $\mathrm{cm}$ by eight $\mathrm{cm}$ and have a plastic platform with ninetysix holes. The boxes hold eight one-inch stubs and six half-inch stubs or thirtytwo half-inch stubs. These contrast with commercially available specimen mount holders which accommodate either four or twelve half-inch stubs and cost at least a couple of dollars each. We find, since availability of the holders is not limiting, that the boxes which are fitted with a removable bottom are preferable to those with a solidly molded casing. We gain easy access to the bottom of these boxes and either insert a packet of 'Drierite' wrapped in tissue to prevent the escape of dust or pour in silicon desiccation beads. Unlike the commercially available holders, these boxes provide a desiccated environment, are available at no cost and accommodate a large number of specimen holders. Our students and faculty are no longer inconvenienced by a variety of stub-holding options such as drilling holes in wood or punching holes in cardboard and storing these holders in assorted containers with a desiccant. Further, specimen shelving allows large numbers of stubs to be easily and efficiently stored in these like-sized boxes. 